\title{
THE EFFECT OF 3D TECHNOLOGIES IN STEREOMETRY TRAINING Penio Lebamovski ${ }^{1}$
}

\begin{abstract}
The software proposed in the report can be used as a technological tool in the teaching and learning of the discipline of stereometry. The aim is to improve the learning process by supporting the development of students' creativity and spatial imagination, qualities needed in the study of spatial geometric bodies. A new boundary method is used in the generation of geometric objects. This new method uses elements of the Cavalieri Indivisible method and Isaac Newton's boundary method, thus achieving higher accuracy with respect to generated objects compared to 3D systems that use the rules of trigonometry in the construction of geometric bodies. This article performs a comparative analysis between a traditional and a new method for generating 3D geometric objects according to certain parameters and criteria. The new method involved in the analysis was proposed by the author of the report. While the traditional one is based on trigonometry. Two parameters were studied, one for accuracy in generating objects, and the second determining speed. In order to generate cylindrical bodies, are used the quadratic Bézier curve and the 3D modeling technique known as an extrusion, which transforms two-dimensional objects into threedimensional ones.. One way to generate a prism and pyramid is by extruding polygons. This report presents a new way of constructing edged bodies. The considered technique for 3D modeling participates in the analysis of the studied methods.
\end{abstract}

UDC Classification: 37.01, DOI: https://doi.org/10.12955/pns.v2.155

Keywords: boundary method, curve, extrusion, stereo system, stereometry, polygon, 3D printing

\section{Introduction}

One of the fastest growing areas of the modern world is 3D technology, which in recent decades has found application in almost all spheres of society, including education. What until recently seemed like science fiction is a reality today. Information technology professionals and educators are actively collaborating to integrate this new technology into the teaching of stereometry. The use of such systems in education will lead to a paradigm shift in the way people view school and university education (Zoneva, 2020). The application of this new technological innovation in education will improve the way students gain new knowledge (Fleck, 2013). Most of the objects that are studied in stereometry are located in 3D space, respectively on the three coordinate axes, which makes it difficult for students who do not have spatial imagination. Therefore, it is necessary to use a stereo system in teaching mathematics, in particular stereometry, in order to develop the creativity and spatial imagination of students, qualities necessary for learning about geometric bodies. The main problem arises when drawing stereometric figures, where unlike planimetry the figures are $2 \mathrm{D}$ and usually the drawing almost $100 \%$ coincides with its original. In three-dimensional space, the figures transferred to a board or white sheet do not coincide with their real image, from where comes the problem that teachers face, namely that the figure in the three dimensions cannot be completely recreated. This problem can be solved with the help of specialized 3D software to generate geometric objects (prism, pyramid, cylinder, cone, sphere, etc.). Also, a comparative analysis of the three most common in Bulgaria and worldwide 3D software systems used for training in stereometry found that only one of them Geogebra (Kmetova, 2015) has a stereoscopic capability, using passive technology. The system presented in this article allows for the implementation of active technology. At the same time, it includes other possibilities that software systems of the scientific literature do not have. Some of the most popular mathematical software are: GeoGebra (Rahman, 2013), Cabri3D (Rososzczuk, 2015) and DALEST (Christou, 2007).

The additional functional possibilities of the new stereoscopic system for training in the discipline of stereometry are:

- Stereoscopic perspective realized through active technology, and future work will include 3D passive technology

- The generated geometric objects are of greater mathematical accuracy than the stereometry training systems that are known so far.

- The student will have the opportunity to make both external and internal inspection of the geometric body

- The system allows you to switch from normal to full screen mode

- It is possible to export objects to a file with the extension .OBJ. In order to print to a 3D printer or add to an augmented reality device

\footnotetext{
${ }^{1}$ Bulgarian Academy of Sciences, Institute of Robotics, Sofia, Bulgaria; penko_13a@abv.bg, p.lebamovski@abv.bg
} 
The use of 3D modeling software applications, including virtual and augmented realities, helps to visually represent complex and abstract shapes that are difficult to be understood by students, who do not have a sufficiently developed spatial imagination. In the teaching process in mathematics (stereometry) the main goals of the teacher are:

- To demonstrate the capabilities of modern technologies through the use of 3D specialized systems

- To acquaint students with how to work with 3D systems in the modeling and processing of geometric objects

- To show the possibilities of VR / AR in solving a geometric problem

With the help of stereometry, students develop their spatial imagination by studying geometric objects in three-dimensional space. The main task of stereometry is to study the position of bodies in threedimensional space. In this way the students get acquainted with the axiomatic methods for presenting geometric objects from stereometry.

The purpose of this article is to present a new stereoscopic system for training in the discipline of stereometry. The system is aimed at middle and high school students studying stereometry from the textbooks of the Bulgarian publishing house "Archimedes". The report performs a comparative analysis and evaluation of a traditional and a new method for generating cylindrical and edged geometric bodies.

\section{Literature review}

\section{CSG (Constructive Solid Geometry)}

The system allows for the representation of geometric objects as solid and wireframe bodies. One of the most used solid models is CSG. After generating a primitive, a Boolean operation can be applied to construct a new primitive. CSG models represent the volumetric body formed by basic primitives (Abdul, 2008). To be used to create this type of models, primitives are appropriately transformed into three-dimensional space (Chen, 2008; Maleshkov, 2014). Transformations can be described by:

- Scaling

- Rotation

- Translation

By using a traditional method, a generated primitive, such as a cube, can be scaled in three dimensions, by $3 \mathrm{D}$ transformation, along one of the axes by a vector. Subsequently, the considered object changes into a parallelepiped. After performing the transformation over the primitive, CSG allows a new type of primitive to be formed through the Boolean operations - union, intersection and difference. Here, methods such as: freeform surface, CSG and sweep, together with the elementary geometric objects, are important for building solid models. In other words, CSG is a collection of elementary geometric objects, transformations and control operations that can be applied to construct 3D models.

\section{Curves / Polygons}

A 3D model is a set of data that is used to display the object in three-dimensional space (Luan, 2008). The information about the object includes data about: pixels, coordinates, size, material, etc. Computer graphics requires 2D and 3D objects to have a numerical description. Objects can be: polygons, curves, mathematical functions and fractals. Polygons are a sequence of connected vertices (Orourke, 2003; 2012). They define a closed area of the plane or space and are bounded by a finite number of segments forming closed curves. The segments of the lines are called edges, and the adjacent points are called vertices. In order to determine the topology when generating a polygon, the traversal of the vertices should be clockwise and counterclockwise (Vince, 2006). Most 3D objects are based on triangles. In some cases, the more triangles there are, the more realistic the object is. By using the detail level technique, the resolution for triangulation of the geometric object can be controlled by the programming language (Klawonn, 2012). Stereometric objects that are part of the stereo system do not need to apply this technique. Exceptions are rotating surfaces. Java3D offers a way to construct surfaces by approximating with polygons. This part of the 3D models, which are generated by triangles, have advantages over the others. At the same time, they are known as triangular meshes. A simple triangular mesh can be, for example, a regular quadrangular pyramid. It consists of four triangles for the surrounding walls and two for the base. It is also called a polygon mesh. When the mesh needs to be modified, it is necessary to clarify the following: 
- What are the six triangles forming the pyramid

- Which two triangles are shared

- Which individuals share common vertices

The triangular mesh can be characterized as a mesh with and without holes (Shirley, 2005). The mathematical representation of the 3D object involves the use of polygons or NURBS (Non-Uniformal Rational Bézier Splines), which are the basic elements of the object (Sabati, 2010). As we said, the polygon is a surface surrounded by at least three vertices (Feng, 2003). The modeling process is a visualization when the polygons are interconnected. The NURBS model is a mathematical description of Bézier curves (Vince, 2006). A 3D model can be created using procedural algorithms. 3D modeling involves the use of polygons or NURBS as the main components of objects. Polygon models are one of the most used in computer graphics. The models are defined as a network of polygons, forming a 3D polyhedron (Orourke, 2012). Each primitive consists of several interconnected ones: vertices, edges, and structural faces. Polygons are relatively easy to define, manipulate and render. They are used in the modeling of complex objects, such as flat surfaces with a large number of polygons. To create cylindrical geometric bodies, quadratic and cubic Bézier curves were used, respectively, in order to generate a circle and extrude it into a cylinder.

The easiest way to control a curve is through a set of points that it can interpolate. In this way the curves are approximate (Shirley, 2005). Here the points influence the shape of the curve and do not determine it precisely. If a set of points needs to be interpolated, their position can be calculated so that the curve passes the interpolation points. The two main types of approximate curves used in computer graphics are: Bézier curves and spline curves (Vince, 2006). The calculation of the control points in the generation of a circle can be done in two ways, namely by quadratic and cubic Bézier curves. When generating a circle using a quadratic curve, the more segments there are, the more accurate the circle is. The Bézier curve is a polynomial curve that approaches its control points. The curves can be polynomial of any degree. For example, the curve of degree $n$ is controlled by $n+1$ control points. Most often the shapes are determined by connecting a number of curves. In computer graphics, the most used curves are the cubic and quadratic Bézier curves, respectively. The curve interpolates the first and last control points by means of zero and one.

There are three main types for defining a curve in mathematics (Shirley, 2005):

1. Implicit: This checks if there is a point in the curve. Usually the implicit representation of a curve is defined by an implicit function (1), so that the curve is a set of points for which the equation is as follows:

$$
f(x, y)=0
$$

2. Parametric: Switch from a free parameter to a set of points. This parameter provides indices of the points on the curve (Dogan, 2017).

3. Generative, procedural: procedures generate the points of the curve.

Finally, we can summarize that the curve is a set of points. The considered representations give us a way to determine these sets of points. Each curve has many possible representations.

\section{Method for modelling three-dimensional objects}

\section{Extrude/Sweep}

The main 3D technique involved in the generation of edged and cylindrical bodies used by most software systems is called extrusion. It can be used to obtain a 3D surface from a $2 \mathrm{D}$ object moved along a given trajectory (Abrams, 2000). This technique is mainly used on polygons and curves. In the sweep / extrude technique, the creation of a volumetric body is done by a plane section and a given rule. The section can be any polygon or a curve formed by three or four control points and a given number of segments. Cubic and quadratic Bézier curves are used to create the stereoscopic application. The rule specifies how to move the two-dimensional object in order to generate a volumetric body. It can be:

- Translation of the section in a given direction

- Rotation of the section around an axis

- Movement of the section in any way

The system uses the first two methods in generating geometric objects. Sweep is a technique in which 2D and 3D objects can be moved through space, while at the same time changing their transformations through: translation, rotation and scaling (Obradovich, 2017). And the movement itself should be done in a certain way. 3D modeling of solid shapes can be performed as procedural modeling. At the same 
time, there are objects that are closely related to mathematical formulas. The procedure itself is a variation of the sweep, where the starting object moves along a way extruding its position in order to generate a solid object. The moving object will change its:

- Position

- Size

- Form

- Transformation

And the end result is the desired solid object. Sweep is a common case of extrusion. The creation of a $3 \mathrm{D}$ object is performed as follows. Initially, there is a $2 \mathrm{D}$ object, which can be either a regular polygon or a complex polygon (Klawonn, 2012). In the process of forming the 3D figure by changing the transformation, the edges of the object must be taken into account. Each edge is actually a simple linear spline. The object can be considered as a group of edges (splines). The object moves through space. When the $2 \mathrm{D}$ figure in the $\mathrm{x} / \mathrm{y}$ plane is extruded by a 3D modeling procedure, moving from the plane to the space (adding the third dimension), the 3D object should appear. This will be a surface if the object is linear or a solid pattern, if it is a closed polygon. In this case, each of the edges of the 2D figure will participate in the construction of the new solid surface. One way to create 3D surfaces is to use the sweep technique to convert a 2D curve through 3D space (Zhang\&Liang, 2007). This can be done by setting a translation vector. The process itself starts from the points of the curve, after applying the rule, the new points are recalculated as part of the 3D surface. Finally, it is necessary to determine the depth for the extrusion itself. The second way to create a 3D surface is to extrude a polygon, which can be two-dimensional or three-dimensional. Again, a rule is applied by translational motion along a certain vector. As with curve extrusion, depth is set here as well.

\section{Results}

Regular polygons are a very important part of 3D programming. The existing traditional ones are characterized by a number of vertices and a radius (Petkov, 2013; Chung, 2009). The report presents new polygons with the characteristic: number of vertices and side. A new boundary method, called "Boundary approach method", is used to generate them. The new boundary method is based on the method of boundaries of Newton (Nyuton, 1989) and indivisible of Cavaleri (Bashmakova, 1975). More complex geometric objects can be constructed on their basis, such as the edged bodies (pyramid and prism). These new polygons are mathematically more accurate than the traditional ones and have a number of advantages. The traditional way to generate polyhedra is by extruding $2 \mathrm{D}$ and $3 \mathrm{D}$ polygons. The method proposed by the author for their generation is more "effective" than the method using the considered 3D technique. The Figure 1 shows a straight and inclined pyramid. The base of which is a regular pentagon. Advantages of the new way of generating polygons and primitives:

1. Mathematically more accurate

2. Easier to learn by students

3. Easy manipulation, as an example can be given the coloring of the walls of a polyhedron

4. Gives the opportunity to perform an internal inspection

5. Stereo effect can be applied by 3D active technology

\section{Suitable for 3D printing}

The most important thing to note is that they can be changed parametrically by the length of the side / edge of the polygon. This will avoid confusion on the part of the students. In the traditional method, in order to increase the side of the polygon, the radius of the inscribed circle must be changed. Therefore, the analysis shows that the objects generated by the new method have better parameters than the objects created in the traditional way by:

1. Polygons generated on the basis of trigonometry rules

2. 3D modeling technique known as "extrusion"

The report performs a comparative analysis between a traditional and a new way of constructing a cylinder. In order to perform the analysis, it is first necessary to draw a circle, after which the 3D modeling technique known as extrusion is applied, which transforms the plane figure into a 3D object. The circle can be generated by quadratic Bézier curves of six segments and three control points for each of them. It should be noted that the quadratic curve is approximate and the more segments there are, the 
more accurate the circle is. Before extruding the geometric object using the system, it is necessary to calculate the coordinate values of the vertices of the curve. For this purpose, the Path2D class from the standard java library and the moveTo quadTo methods are used. Figure 2 shows the control points of the curves. Figure 3 shows a straight and inclined cylinder. The base of which is generated by a square curve The calculation of the coordinate values of the vertices of the curves can be done in two ways: By applying a traditional method using trigonometry or by applying the new boundary method called the "boundary approach method"

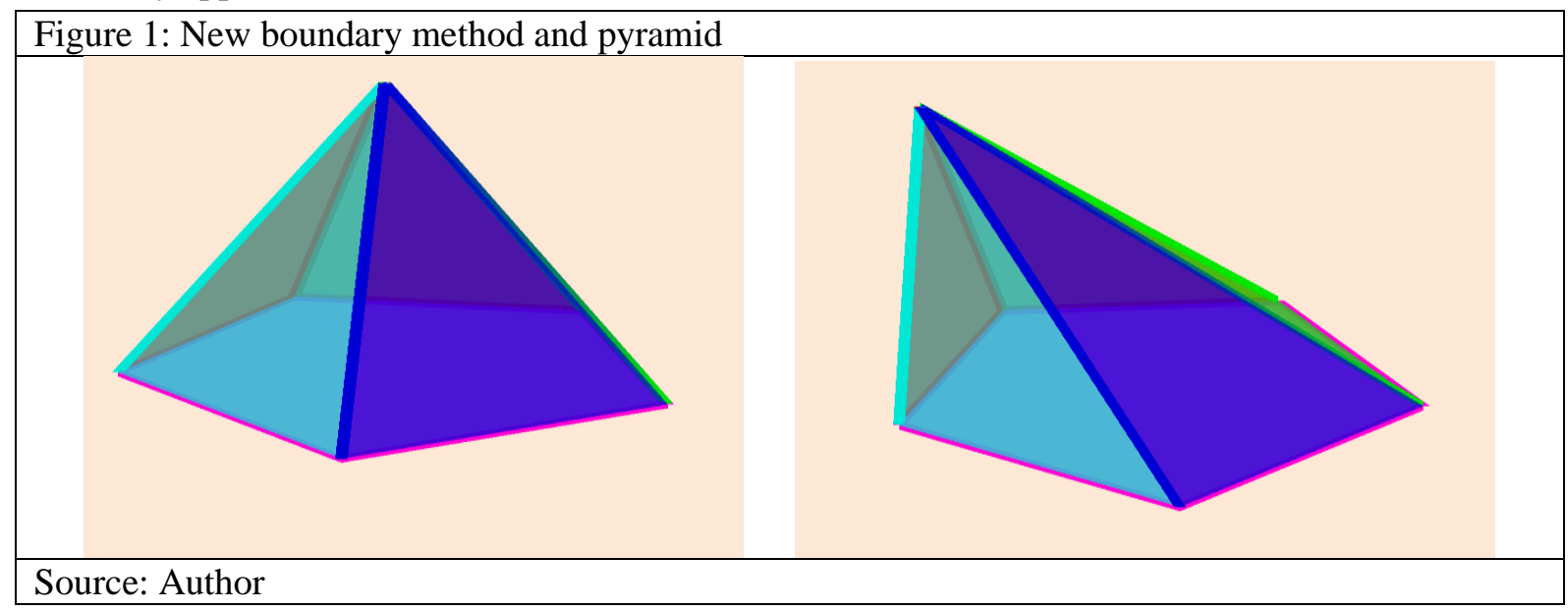

To generate a circle, using the traditional method (2), (3) it is necessary to perform the following:

1. Set the radius of the inscribed circle

2. Set the number of segments and calculate the center angle $=\alpha$

3. Calculate the cosine and sine of the central angle

4. Calculate the radius of the circumscribed circle

5. Calculation of even and odd points by formula

After creating the circle, it is extruded to a 3D cylinder. The following basic steps are required:

1. The circle is located on the coordinate axis along the four quadrants

2. The control points are defined and calculated

3. The first vertex can be chosen arbitrarily, and must participate in the generation of the first and last segment

Figure 4 shows a piece of code involved in generating a circle using the new method

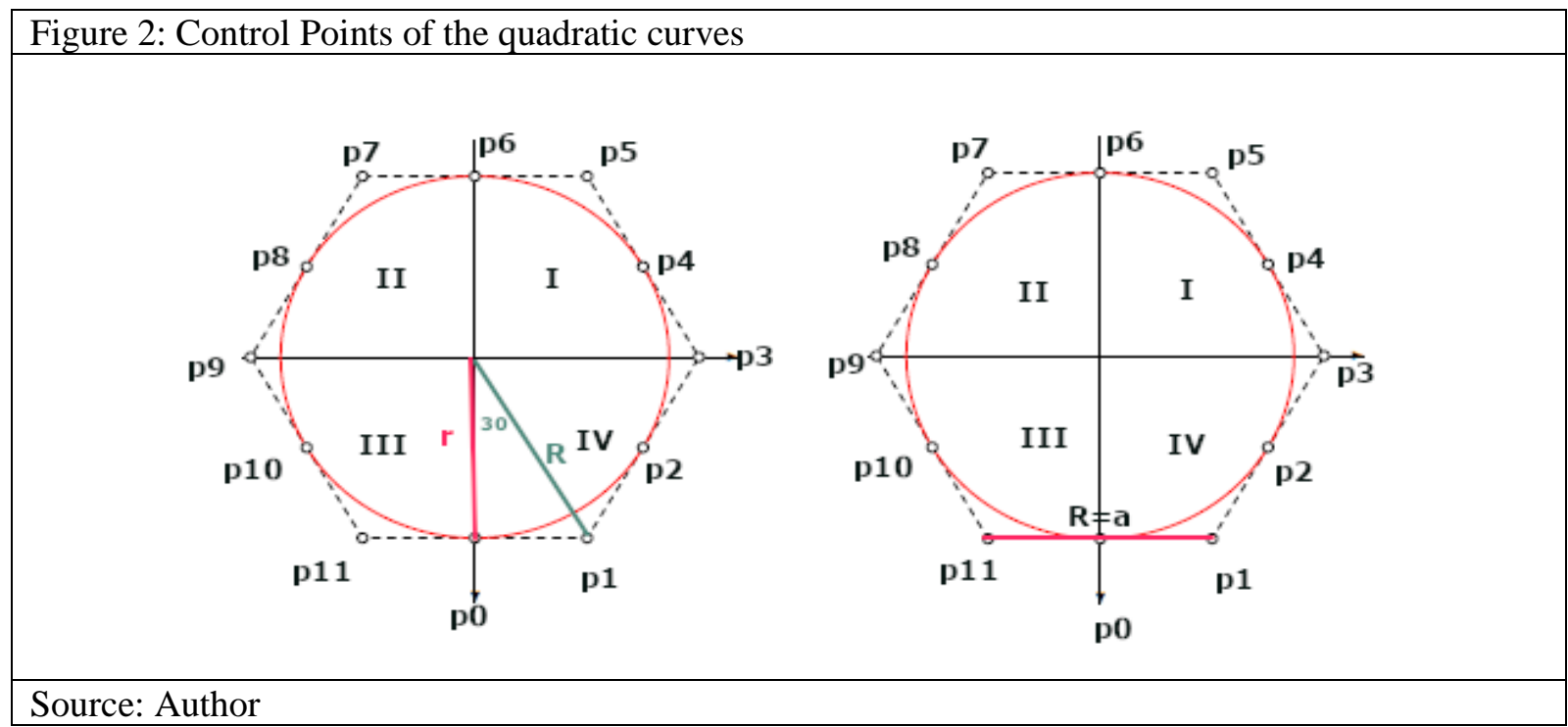

To determine the coordinate values of the vertices of the six-segment Bézier quadratic curve, the proposed new method uses only the dependence relation of parallel line segments. That is what makes the new method different from the traditional one, which uses trigonometry (sine, cosine and radius). Before calculating the coordinates of the vertices, it is necessary to determine by the new method the 
ratio of the parallel line segments in an isosceles trapezoid which are 1:3/2:2. While by means of the traditional method the considered 5 parameters are needed. The analysis is performed on the basis of the parameter speed and "simplified" calculations, which are in favor of the new boundary method. double $\mathrm{r}=($ double $)(\mathrm{a} / 2)^{*} \mathrm{sqrt}(3)$; The specified processing time when using the new method is $10 \%$ less than the traditional one. To determine the vertices of the quadric curve when applying the traditional method, the following conditions are applied (Reimers, 2011):

$$
\begin{gathered}
P_{2 i}=\left(\begin{array}{c}
+r \cdot \sin (2 i \cdot \alpha) \\
-r \cdot \cos (2 i \cdot \alpha)
\end{array}\right) \text { за } i=0 \ldots . n . \\
P_{2 i+1}=\left(\begin{array}{c}
+R \cdot \sin ((2 i+1) \cdot \alpha) \\
-R \cdot \cos ((2 i+1) \cdot \alpha)
\end{array}\right) \text { за } i=0 \ldots . n-1 .
\end{gathered}
$$
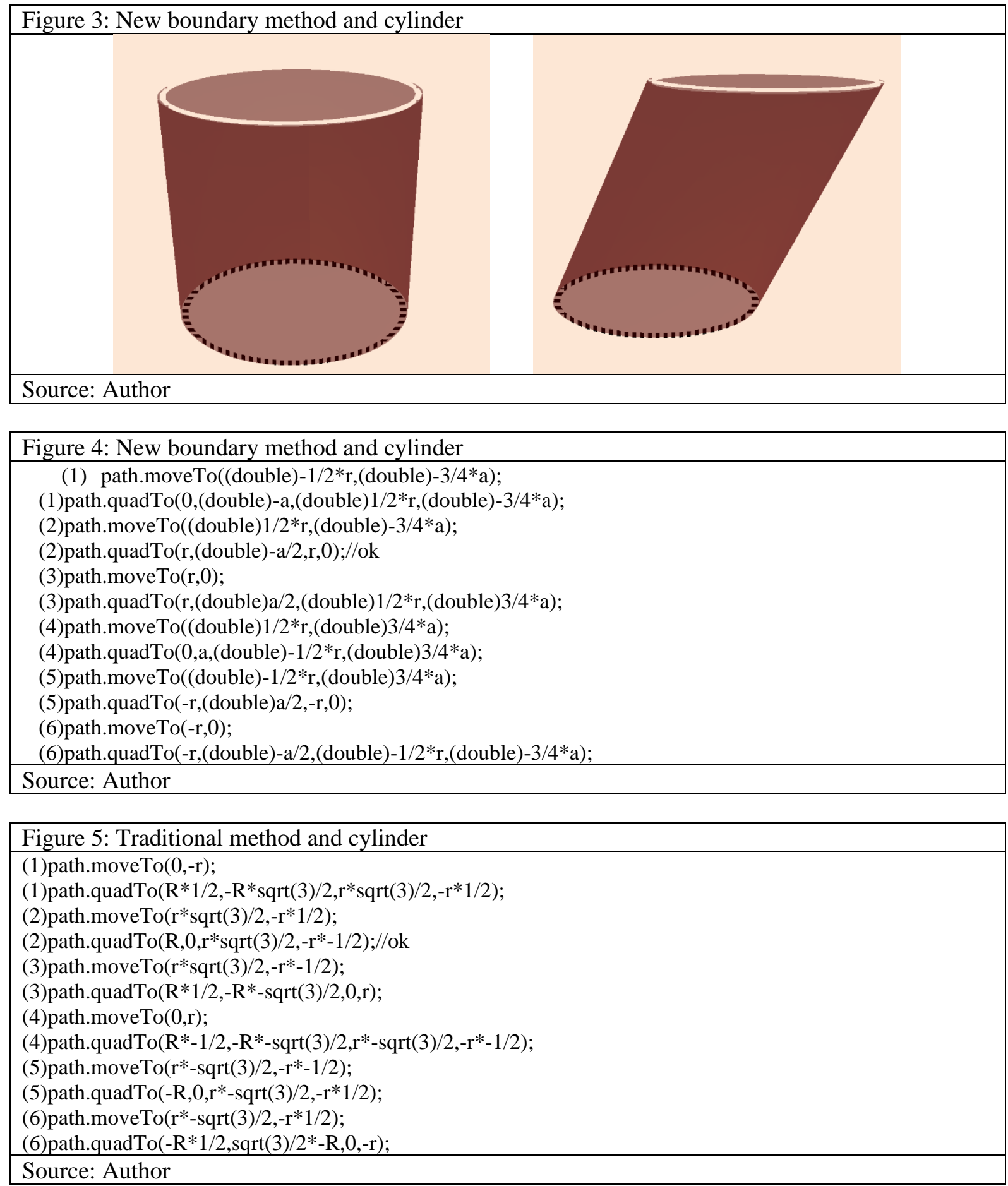


\section{Conclusion}

Today, with the help of 3D technology, the process of training stereometry can be greatly facilitated. The use of appropriate software would enhance creativity and spatial imagination, qualities needed in stereometry training. The report presents a new method for generating cylindrical and edged bodies. From the performed comparative analysis between the traditional and the new way of generating geometric objects it was established that the new approach has better indicators. Which shows that teaching stereometry with the new software would be more effective than software programs known in the scientific literature. The stereoscopic system is very suitable for high school students. The interface of the system is as simple, easy and convenient to use as possible. Future work will be focused on testing the system with real users. Additionally, future work will improve the software`s interface as well as focus on the application of the system in other scientific fields, such as: history, astronomy and medicine.

\section{References}

Abdul, R. (2008). Spatiel Data Modelling for 3D GIS. Berlin Heidelberg: Springer-Verlag.

Abrams, S., Allen, P. (2000). Computing swept volumes. Visualisation and Computer Animation, 69-82.

Bashmakova, I. (1975). Istoria nd metematikata[History of mathematics]. Sophia: Izdatelstvo nauka i izkustvo.

Chen, J., Chen,C. (2008). Foundations of 3D Graphics Programming Using JOGL and Java3D. London: Springer.

Christou, C., Sendova, C., Matos, J. (2007). Stereometry Activities with Dalest. Nicozia.

Chung, C, Cheng,C. (2009). Interactive Web-Based Virtual Reality with Java3D. New York.

Dogan, F., Yayli, Y. (2017). The relation between parameter curves and lines of curvature on canal surfaces. Kuwait Journal, 29-35.

Feng, Y., Owen, D. (2003). A 2D polygon/polygon contact model:algorithmic aspects. Emerald.

Fleck, S., G.S. (2013). An augmented Reality Environment for Astronomy Learning in Ele;entary Grades. 25th Conference on LInteraction Home -Machine.

Klawonn, F. (2012). Introduction to Computer Graphics Using Java 2D and 3D. Springer.

Kmetova, M. (2015). Rediscovered Anaglyph in program GeoGebra.

Luan, Xi., Xie, Yu., Ying, L. (2008). Research and Development of 3D Modelling. IJCSNS International Journal of Computer Science and Network.

Maleshkov, S. G. (2014). Komputarna graphika i photorelistichna vizualizacia/Computer graphics and photorealistic vizualization]. Sophia: Nov Bulgarski Universitet.

Nyuton, I. (1989). Matematicheskie nachala naturalna philosophia[Mathematical principles natural philosophy]. Moskva: Izdatelstvo Nauka.

Obradovich, M. (2017). Creating 3D shapes by time extrusion of moving objects. Conference 20 Generative Art Conference GA 2017.

Orourke, J. (2003). Principles of the Three-Demensional Computer Animation. W.W. North\&Company.

Orourke, J. (2012). Computational Geometry in C. Cambridge University Press.

Petkov, E. (2013). Osnovi na kompyutarnata graphika [Basics of computer graphics]. Veliko Tarnovo: Faber.

Rahman, M, Puteh, M. (2013). Learning trigonometry using GeoGebra learning module: Are under achieve pupils motivated. AIP Conference Proceedings Vol. 1750.

Reimers, D. (2011). Bezier Curves An introduction.

Rososzczuk, R. (2015). Application of Cabri3D in teaching stereometry. Advances inScience and Technology Research Journal, Vol.9.

Sabati, Z. N. (2010). Standardizatation of 3D Models Creation procedures in Computer Graphics. International Design Conference. Dubrovnik.

Shirley, P. (2005). Fundamentals of Computer Graphics. Utah.

Vince, J. (2006). Mathematics for Computer Graphics. London: Springer.

Yeh, A. N. (2004). Towards a semiotic framework for using technology in mathematics education: the case of learning 3D geometry. International Conference on Computers in Education. Melbourne.

Z., Hong, Liang,Y. (2007). Computer Graphics using Java2D and Java3D. Pearson Education.

Zoneva, L. (2020). Degree of Digital technological Integration in The Bulgarian secondary technological education. $C B U$ International Conference on innovations in Science and Education. Prague. 